\title{
Stability and stabilization of linear positive systems on time scales
}

\section{Zbigniew Bartosiewicz ${ }^{1}$ D}

Received: 17 May 2019 / Accepted: 10 January 2020 / Published online: 28 January 2020

(c) The Author(s) 2020

\begin{abstract}
It is shown that a positive linear system on a time scale with a bounded graininess is uniformly exponentially stable if and only if the characteristic polynomial of the matrix defining the system has all its coefficients positive. Then this fact is used to find necessary and sufficient conditions of positive stabilizability of a positive control system on a time scale.
\end{abstract}

Keywords Positive linear system · Positive stability $\cdot$ Positive stabilization

Mathematics Subject Classification 93D05 - 93D15

\section{Introduction}

In positive systems the state variables take nonnegative values. Such systems appear in biology, medicine and economics [1,2]. We study here linear positive systems on time scales. A time scale is a model of time. Time may be continuous, discrete or mixed-partly continuous and partly discrete. The delta derivative, which is used in delta differential equations that model positive systems on time scales, may be equal to ordinary derivative or may be equal to a difference quotient, depending on the time scale and a particular point (see Appendix for the precise definitions).

It is known that in the continuous-time case the positive system $\dot{x}=A x$ is exponentially stable (at 0 ) if and only if the characteristic polynomial of the matrix $A$, $\chi_{A}(\lambda)=\operatorname{det}(\lambda I-A)$, has all its coefficients positive (see $\left.[1,2]\right)$. We show that this is true for a system on an arbitrary time scale as long as the graininess function of

This work has been supported by the Bialystok University of Technology Grant No. S/WI/00/2020.

Zbigniew Bartosiewicz

z.bartosiewicz@pb.edu.pl

1 Faculty of Computer Science, Bialystok University of Technology, Wiejska 45A, 15-351 Białystok, Poland 
the time scale (which measures the distance between a particular time instant and the next instant) is bounded and uniform exponential stability is considered. Thus any discretization (in particular nonuniform) of a positive continuous-time uniformly exponentially stable system that preserves positivity gives an uniformly exponentially stable system. We rely here on $[3,4]$, where uniform exponential stability of linear systems was studied. Stability of linear systems on time scales was also considered in [5-14]. Various authors considered different concepts of stability and different variants of the same concept. For example, in the definition of exponential stability, to estimate the solutions, either the standard exponential function was used or the exponential function on the time scale. Relations between asymptotic stability and exponential stability for continuous-time systems were studied in [15].

If a control system is not uniformly exponentially stable, we can try to use feedback to stabilize the system. If our system is positive, it is natural to require that the feedback preserves positivity. This procedure is called positive stabilization. We give necessary and sufficient conditions for positive stabilizability of positive systems on time scales with bounded graininess. One of the conditions is equivalent to standard stabilizability of a system on a time scale. The other consists of inequalities that must be satisfied by the matrix that defines the feedback.

Stabilizability of positive linear systems were investigated in e.g. [1,2], separately for continuous-time and discrete-time systems. Other problems for positive linear systems on time scales like reachability, observability and realizations were studied in [16-21].

\section{Preliminaries}

We shall study control systems on time scales. A short introduction to the calculus on time scales is provided in the appendix. More information can be found in e.g. [22].

Let $\mathbb{T}$ be a time scale with the forward graininess $\mu_{\mathbb{T}}$. We shall assume that $\sup \mathbb{T}=$ $+\infty$ and denote $\mu_{\mathbb{T}}:=\sup \left\{\mu_{\mathbb{T}}(t): t \in \mathbb{T}\right\}$.

We shall write $e_{q}\left(t, t_{0}\right)$ to denote the generalized exponential function of $q$, initialized at $t_{0}$ and evaluated at $t$. It is the unique forward solution $\left(t \geq t_{0}\right)$ of the initial value problem

$$
x^{\Delta}(t)=q(t) x(t), x\left(t_{0}\right)=1,
$$

where $x^{\Delta}(t)$ means the delta derivative of the function $x$ at time $t \in \mathbb{T}$.

For a square matrix $A$ with complex or real elements, $\operatorname{spec}(A)$ will mean its spectrum, i.e. the set of its eigenvalues and $\|A\|$ will mean the standard spectral norm of A.

Let $A$ be a real $n \times n$ matrix. Consider the time-invariant linear system on the time scale $\mathbb{T}$

$$
x^{\Delta}(t)=A x(t),
$$

where $x(t) \in \mathbb{R}^{n}$ and $t \in \mathbb{T}$. 
Theorem 1 ([22]) Let $t_{0} \in \mathbb{T}$ and $x_{0} \in \mathbb{R}^{n}$. Then system (1) with the initial condition $x\left(t_{0}\right)=x_{0}$ has a unique solution $x:\left[t_{0},+\infty\right) \cap \mathbb{T} \rightarrow \mathbb{R}^{n}$.

This result can be extended to matrix-valued solutions of (1).

Let $t_{0} \in \mathbb{T}$ and let $X:\left[t_{0},+\infty\right) \cap \mathbb{T} \rightarrow \mathbb{R}^{n \times n}$ satisfy the matrix delta differential equation

$$
X^{\Delta}(t)=A X(t)
$$

and the initial condition $X\left(t_{0}\right)=I$, where $I$ is the $n \times n$ identity matrix. Its value at $t \in \mathbb{T}, t \geq t_{0}$, is denoted by $e_{A}\left(t, t_{0}\right)$. , Then the solution of the initial value problem

$$
x^{\Delta}=A x, x\left(t_{0}\right)=x_{0}
$$

can be written as

$$
x(t)=e_{A}\left(t, t_{0}\right) x_{0} .
$$

Thus $e_{A}\left(t, t_{0}\right)$ is the transfer matrix of the system $x^{\Delta}=A x$ in the language of ordinary differential equations.

All the definitions and statements of this section can be naturally extended to complex-valued functions and matrices.

\section{Positive control systems on time scales}

Let $\mathbb{R}_{+}^{n}$ mean the set of all vectors from $\mathbb{R}^{n}$ with nonnegative components.

Consider now a linear control system on a time scale $\mathbb{T}$ :

$$
x^{\Delta}(t)=A x(t)+B u(t)
$$

where $t \in \mathbb{T}, x(t) \in \mathbb{R}^{n}$ and $u(t) \in \mathbb{R}^{m}$. We assume that the control $u$ is a piecewise continuous function of time.

Definition 1 The system (3) is positive if for all $t_{0} \in \mathbb{T}$ and any control $u:\left[t_{0}, t_{1}\right]_{\mathbb{T}} \rightarrow$ $\mathbb{R}_{+}^{m}$ the trajectory starting from any $x\left(t_{0}\right)=x_{0} \in \mathbb{R}_{+}^{n}$ and corresponding to $u$ stays in $\mathbb{R}_{+}^{n}$ for all $t \in\left[t_{0}, t_{1}\right]_{\mathbb{T}}$.

We extend $\mathbb{R}$ adding $+\infty: \overline{\mathbb{R}}:=\mathbb{R} \cup\{+\infty\}$. For $a \in \mathbb{R}$ we set $a+\infty:=+\infty$, $1 / 0=+\infty, 1 /+\infty=0$ and $+\infty>a$.

Let $\gamma:=\gamma(\mathbb{T}):=1 / \bar{\mu}_{\mathbb{T}}$. Then $\gamma(\mathbb{R})=+\infty, \gamma(h \mathbb{Z})=1 / h$ and $\gamma\left(2^{\mathbb{N}}\right)=0$.

Let $A_{\mathbb{T}}:=A+\gamma(\mathbb{T}) I$, where $\gamma(\mathbb{T}) I$ is the diagonal matrix with $\gamma(\mathbb{T})$ on the diagonal.

Proposition $1([4,17])$ The system $x^{\Delta}=A x+B u$ is positive if and only if all elements of $A_{\mathbb{T}}$ and $B$ are nonnegative. 
The condition that $A_{\mathbb{T}}$ is nonnegative implies that $A$ is a Metzler matrix, i.e. its off-diagonal elements are nonnegative. Let $M$ be an $n \times n$ Metzler matrix. Let us set $c(M):=\min \{a \geq 0: A+a I \geq 0\}$. From the definition of $c(M)$ we get:

Proposition $2 A_{\mathbb{T}}$ is nonnegative if and only if $A$ is Metzler and $c(A) \leq \gamma(\mathbb{T})$.

For a real $n \times n$ matrix $M$ let $r(M):=\max \{|\lambda|: \lambda \in \operatorname{spec}(M)\}$ be the spectral radius of $M$ and $\eta(M):=\max \{\operatorname{Re} \lambda: \lambda \in \operatorname{spec}(M)\}$ be the spectral abscissa.

Theorem 2 (Perron-Frobenius) Let $P \in \mathbb{R}_{+}^{n \times n}$. Then $r(P)$ is an eigenvalue of $P$.

The proof of Theorem 2 can be found e.g. in [23].

Proposition 3 Let $M$ be a real $n \times n$ Metzler matrix. Then $\eta(M)=r(M+\alpha I)-\alpha$ for all $\alpha \geq c(M)$, and $\eta(M)$ is an eigenvalue of $M$.

The proof of Theorem 2 can be found e.g. in [4]. One needs here the useful property of spectrum:

$$
\operatorname{spec}(M+\alpha I)=\operatorname{spec}(M)+\alpha,
$$

where $M$ is a square matrix. It will be used later in our investigations.

\section{Stability}

We consider here uniform exponential stability of the equilibrium point 0 of a linear system on a time scale. This property will be shortly called uniform exponential stability of the system.

Definition 2 ([3]) System $x^{\Delta}=A x$ is uniformly exponentially stable if there are constants $K \geq 1$ and $\alpha>0$, and an open neighborhood $V$ of 0 in $\mathbb{R}^{n}$ such that for every $t_{0}, t \in \mathbb{T}$ with $t \geq t_{0}$ and every $x_{0} \in \mathbb{R}^{n} \cap V$, the forward trajectory $x$ of the system, corresponding to the initial condition $x\left(t_{0}\right)=x_{0}$, satisfies $\|x(t)\| \leq$ $K \exp \left(-\alpha\left(t-t_{0}\right)\right)\left\|x_{0}\right\|$.

It is known that the condition $\sup \left\{\mu_{\mathbb{T}}(t): t \in \mathbb{T}\right\}<+\infty$ is necessary for uniform exponential stability of the system $x^{\Delta}=f(x)$ (see e.g. [24]). Therefore, from now on we shall assume that $\bar{\mu}_{\mathbb{T}}:=\sup \left\{\mu_{\mathbb{T}}(t): t \in \mathbb{T}\right\}<+\infty$.

For linear systems on time scales uniform exponential stability has been well investigated. The following proposition follows directly from the definition.

Proposition 4 ([3]) The following conditions are equivalent:

(i) system $x^{\Delta}=A x$ is uniformly exponentially stable,

(ii) there exist constants $\alpha>0$ and $K \geq 1$ such that for all $t_{0} \in \mathbb{T}$

$$
\left\|e_{A}\left(t, t_{0}\right)\right\| \leq K e^{-\alpha\left(t-t_{0}\right)}
$$

for all $t \in \mathbb{T}$ such that $t \geq t_{0}$. 
We will say that $\lambda \in \mathbb{C}$ is uniformly exponentially stable with respect to $\mathbb{T}$ if the scalar equation on $\mathbb{T}$

$$
x^{\Delta}=\lambda x,
$$

where $x \in \mathbb{C}$, is uniformly exponentially stable.

The set of all uniformly exponentially stable $\lambda \in \mathbb{C}$ with respect to $\mathbb{T}$ will be denoted by $\mathcal{S}_{\mathbb{T}}$. It depends on the time scale $\mathbb{T}$. For $\mathbb{T}=\mathbb{R}$ it is equal to $\mathbb{C}_{-}$, while for $\mathbb{T}=h \mathbb{Z}$ it is the open disc of the radius $1 / h$ with the center $-1 / h$. But for other time scales such sets are often unknown. More details can be found in $[3,13]$.

In [4] the following has been shown:

Theorem 3 Let $\mu(\mathbb{T})<+\infty$. Then $\mathcal{S}_{\mathbb{T}} \subset \mathbb{C}_{-}$and for $\gamma=\gamma(\mathbb{T})<+\infty$ the set $\mathcal{S}_{\mathbb{T}}$ contains the open disc of radius $\gamma$ centered at $-\gamma$. For $\gamma(\mathbb{T})=+\infty, \mathcal{S}_{\mathbb{T}}=\mathbb{C}_{-}$.

For linear systems uniform exponential stability can be characterized by the eigenvalues of the matrix $A$.

Theorem 4 ([3]) The following conditions are equivalent:

(i) system $x^{\Delta}=A x$ is uniformly exponentially stable,

(ii) every $\lambda \in \operatorname{spec}(A)$ is uniformly exponentially stable with respect to $\mathbb{T}$.

Corollary 1 System $x^{\Delta}=$ Ax is uniformly exponentially stable if and only if $\operatorname{spec}(A) \subset$ $\mathcal{S}_{\mathbb{T}}$.

For positive systems we are interested in what happens with the trajectories that start from initial states belonging to $\mathbb{R}_{+}^{n}$. This leads to the following definition.

Definition 3 Assume that system $x^{\Delta}=A x$ is positive. We say that $x^{\Delta}=A x$ is positively uniformly exponentially stable if there are constants $K \geq 1$ and $\alpha>0$, and an open neighborhood $V$ of 0 in $\mathbb{R}^{n}$ such that for every $t_{0}, t \in \mathbb{T}$ with $t \geq t_{0}$ and every $x_{0} \in \mathbb{R}_{+}^{n} \cap V$, the forward trajectory $x$ of the system, corresponding to the initial condition $x\left(t_{0}\right)=x_{0}$, satisfies $\|x(t)\| \leq K \exp \left(-\alpha\left(t-t_{0}\right)\right)\left\|x_{0}\right\|$ for all $t \in \mathbb{T}$ such that $t \geq t_{0}$.

It appears that for linear positive systems both properties coincide.

Proposition 5 ([25]) A positive system $x^{\Delta}=$ Ax is positively uniformly exponentially stable if and only if it is uniformly exponentially stable.

Proposition 5 implies that for positive linear systems positive uniform exponential stability can be characterized with the aid of the spectrum of the matrix $A$. However this spectrum for a positive system has a specific structure, so other tools for checking (positive) uniform exponential stability can be employed.

Theorem 5 A linear positive system $x^{\Delta}=A x$ is positively uniformly exponentially stable if and only if all the coefficients of the characteristic polynomial $\chi_{A}(\lambda)=$ $\operatorname{det}(\lambda I-A)$ of the matrix A are positive. 
Proof Necessity. Assume that the system $x^{\Delta}=A x$ is positively uniformly exponentially stable. From Proposition 5, Theorem 4 and Theorem 3 it follows that all eigenvalues $\lambda_{1}, \ldots, \lambda_{n}$ of $A$ have negative real parts. Then

$$
\chi_{A}(\lambda)=\left(\lambda-\lambda_{1}\right) \cdots\left(\lambda-\lambda_{n}\right)
$$

If $\lambda_{i}$ is complex then $\lambda_{i}=-a+b i$ for $a>0$. Since $A$ is real, $\lambda_{j}=-a-b i$ for some $j \neq i$. Then the polynomial $\left(\lambda-\lambda_{i}\right)\left(\lambda-\lambda_{j}\right)=\lambda^{2}+2 a \lambda+b^{2}$ has all its coefficients positive. If $\lambda_{k}$ is real, then $\lambda_{k}=-c$ for $c>0$, so the polynomial $\lambda-\lambda_{k}=\lambda+c$ has all its coefficients positive as well. Since $\chi_{A}(\lambda)$ is a product of polynomials of these two types, it also has all its coefficients positive.

Sufficiency. Let us assume that $\chi_{A}(\lambda)=\lambda^{n}+a_{n-1} \lambda^{n-1}+\cdots+a_{0}$ has all its coefficients positive. Since $A$ is a Metzler matrix, from Proposition 3 it follows that $\lambda_{1}:=\eta(A) \in \mathbb{R}$ is an eigenvalue of $A$. If it were nonnegative, then $\chi_{A}\left(\lambda_{1}\right)$ would be greater than 0 , and this would contradict the fact that $\lambda_{1}$ is an eigenvalue of $A$. Thus $\eta(A)<0$, which means that all eigenvalues of $A$ have negative real parts. Assume now that $\gamma(\mathbb{T})<+\infty$. Since $c(A) \leq \gamma(\mathbb{T})$ (from positivity of the system and Proposition 2), using again Proposition 3 we get

$$
r(A+\gamma(\mathbb{T}) I)=\eta(A)+\gamma(\mathbb{T})<\gamma(\mathbb{T}),
$$

which implies that $\operatorname{spec}(A+\gamma(\mathbb{T}) I)$ is contained in the disc of radius $\gamma(\mathbb{T})$ centered at 0 . By $(4), \operatorname{spec}(A)=\operatorname{spec}(A+\gamma(\mathbb{T}) I)-\gamma(\mathbb{T})$, so $\operatorname{spec}(A)$ is contained in in the disc of radius $\gamma(\mathbb{T})$ centered at $-\gamma(\mathbb{T})$. From Theorem 3 we get that $\operatorname{spec}(A) \subset \mathcal{S}_{\mathbb{T}}$, which, by Corollary 1 , implies that the system is uniformly exponentially stable. For $\gamma(\mathbb{T})=+\infty, \mathcal{S}_{\mathbb{T}}=\mathbb{C}_{-}, \operatorname{so} \operatorname{spec}(A) \subset \mathcal{S}_{\mathbb{T}}$ as well.

Remark 1 This fact has long been known for continuous-time systems [1,2], when $\mathbb{T}=\mathbb{R}$. For a discrete-time positive system of the form

$$
x(k+1)=\tilde{A} x(k)
$$

(uniform) exponential stability has been characterized by the condition that the characteristic polynomial of $A:=\tilde{A}-I$ has positive coefficients [1,2]. But (7) is equivalent to the system on the time scale $\mathbb{T}=\mathbb{Z}$ :

$$
x^{\Delta}(k)=x(k+1)-x(k)=A x(k)
$$

so this characterization of uniform exponential stability agrees with the characterization presented in Theorem 5 for systems on arbitrary time scales with the bounded graininess. In particular, this characterization is valid for $\mathbb{T}=h \mathbb{Z}$, where $h>0$. Consider an Euler discretization of the positive continuous-time system $\dot{x}=A x$ with the step $h$ :

$$
x^{\Delta}(k h):=\frac{x((k+1) h)-x(k h)}{h}=A x(k h) .
$$


Since the matrix $A$ is the same for both systems, from Theorem 5 we conclude that uniform exponential stability of the continuous-time system implies uniform exponential stability of the discretized system, provided the latter is positive. This holds if and only if $h \leq 1 / c(A)$.

\section{Stabilization}

The main goal of this section is to find conditions for feedback stabilization of a positive linear system on a time scale $\mathbb{T}$ :

$$
\Sigma: x^{\Delta}=A x+B u,
$$

with $x(t) \in \mathbb{R}^{n}, u(t) \in \mathbb{R}^{m}$. However we want to preserve positivity of the system.

Definition 4 System (8) is positively stabilizable if there is a feedback $u=K x$, such that the closed-loop system $x^{\Delta}=(A+B K) x$ is positive and positively uniformly exponentially stable.

Remark 2 We do not assume that $K$ is nonnegative, so $u=K x$ may have negative components, even for $x \in \mathbb{R}_{+}^{n}$. This may be interpreted as enlarging the set of admissible control values and making it depend on the state $x$. But we still have nonnegativity of trajectories of the system that start from the points of $\mathbb{R}_{+}^{n}$ and correspond to controls defined by the feedback. A good example of such situation is a compartmental system (see e.g. [1,2]). Replacing input $u$, which is interpreted as the inflow rate of material to a compartment, by the feedback $K x$ with negative $K$, we allow for reducing the amount of material in the compartment. This can help stabilize the system. The closed-loop system is still positive.

On the other hand, if the open-loop system is not positively uniformly exponentially stable, then by applying the feedback $u=K x$ with a nonnegative $K$ we preserve positivity of the system, but we cannot stabilize it (see [2]).

Since positive stabilizability implies usual stabilizability (where we do not require that the closed-loop system is positive), we have the following:

Proposition 6 If the positive system (8) is positively stabilizable, then the following condition holds:

$$
\forall \lambda \in \operatorname{spec}(A): \lambda \notin S(\mathbb{T}) \Rightarrow \operatorname{rank}[\lambda I-A, B]=n
$$

Condition (9) is equivalent to standard stabilizability [10], i.e. it does not guarantee existence of $K$ such that the system $x^{\Delta}=(A+B K) x$ is uniformly exponentially stable and positive at the same time. It exploits Popov-Belevitch-Hautus criterion of controllability of a linear system (see e.g. [26]).

To find necessary and sufficient condition for positive stabilizability we need to add another condition to (9). For simplicity we assume now that $m=1$, so $B=b=$ $\left(b_{1}, \ldots, b_{n}\right)^{T}, K=\left(k_{1}, \ldots, k_{n}\right)$ and (8) becomes now 


$$
\Sigma: x^{\Delta}=A x+b u,
$$

and (9) becomes

$$
\forall \lambda \in \operatorname{spec}(A): \lambda \notin S(\mathbb{T}) \Rightarrow \operatorname{rank}[\lambda I-A, b]=n
$$

Let us define for $j=1, \ldots, n$ :

$$
\alpha_{j}:= \begin{cases}-\infty, & \text { if } \gamma(\mathbb{T})=+\infty \text { and for every } \\ \max _{i \neq j, b_{i} \neq 0}\left\{\frac{-a_{i j}}{b_{i}}\right\}, & i \neq j, b_{i}=0 \\ \max \left\{\max _{i \neq j, b_{i} \neq 0}\left\{\frac{-a_{i j}}{b_{i}}\right\}, \frac{-a_{j j}-\gamma(\mathbb{T})}{b_{j}}\right\}, & \text { if } b_{j}=0 \text { or } \gamma(\mathbb{T})=+\infty \text { and } \\ & \text { there is } i \neq j \text { such that } b_{i} \neq 0\end{cases}
$$

Observe that $\alpha_{j} \leq 0$ for any $j=1, \ldots, n$.

Now assume that $\operatorname{rank}\left[b, A b, \ldots, A^{n-1} b\right]=s$. Then $A^{s} b=-a_{1} b-\cdots-a_{s} A^{s-1} b$ for some $a_{1}, \ldots, a_{s} \in \mathbb{R}$. Let us define a basis of $\mathbb{R}^{n}$ as follows.

$v_{s-i}=A^{i} b+a_{s} A^{i-1} b+\cdots+a_{s-i+1} b$ for $i=0, \ldots, s-1$, and, if $s<n$, $v_{s+1}, \ldots, v_{n}$ chosen arbitrarily so that $v_{1}, \ldots, v_{n}$ are linearly independent. In particular, $v_{s}=b$.

Then for $i=0, \ldots, s-2, A v_{s-i}=v_{s-i-1}-a_{s-i} b$ and $A v_{1}=a_{1} b$. Thus, letting $T=\left(v_{1}, \ldots, v_{n}\right)$ we get

$$
\tilde{A}:=T^{-1} A T=\left(\begin{array}{cccccc}
0 & 1 & 0 & \cdots & 0 & * \\
0 & 0 & 1 & \cdots & 0 & * \\
\vdots & \vdots & \vdots & \ddots & \vdots & \vdots \\
0 & 0 & 0 & \ldots & 1 & * \\
-a_{1} & -a_{2} & -a_{3} & \cdots & -a_{s} & * \\
0 & 0 & 0 & \ldots & 0 & A_{22}
\end{array}\right), \tilde{b}:=T^{-1} b=\left(\begin{array}{c}
0 \\
0 \\
\vdots \\
0 \\
1 \\
0
\end{array}\right)
$$

where $A_{22}$ is a $(n-s) \times(n-s)$ matrix and 0's in the last rows of $\tilde{A}$ and $\tilde{b}$ mean zero $(n-s) \times 1$ matrices.

Now we can state a characterization of positive stabilizability of a positive system.

Theorem 6 The positive system (10) is positively stabilizable if and only if condition (11) is satisfied and the following set of linear inequalities for $K=\left(k_{1}, \ldots, k_{n}\right)$ is consistent:

$$
k_{j} \geq \alpha_{j}, j=1, \ldots, n, K v_{i}<a_{i}, i=1, \ldots, s .
$$

Proof Necessity. From Proposition 6 it follows that condition (11) is necessary for positive stabilizability of system (10). Moreover, positive stabilizability implies existence of $K=\left(k_{1}, \ldots, k_{n}\right)$ such that the closed-loop system $x^{\Delta}=(A+b K) x$ is positive. Thus such $K$ must satisfy $a_{i j}+b_{i} k_{j} \geq 0$ for $i \neq j$ and $a_{j j}+b_{j} k_{j}+\gamma(\mathbb{T}) \geq 0$ for $j=1, \ldots, n$. If $\gamma(\mathbb{T})=+\infty$, the second inequality is always satisfied. Easy calculation shows that these inequalities are equivalent to the inequalities $k_{j} \geq \alpha_{j}$, $j=1, \ldots, n$. To show that the inequalities $K v_{i} \leq a_{i}$ are also necessary let us 
transform system (10) to $\tilde{x}^{\Delta}=\tilde{A} \tilde{x}+\tilde{b} u$, where $\tilde{A}$ and $\tilde{b}$ are given by (12). Positive stabilizability of system (10) implies existence of $K$ such that $\chi_{A+b K}$ has positive coefficients. This is equivalent to existence of $\tilde{K}=K T$ such that $\chi_{\tilde{A}+\tilde{b} \tilde{K}}$ has positive coefficients (since $\tilde{A}+\tilde{b} \tilde{K}=T^{-1}(A+b K) T$ and thus the characteristic polynomials of $\tilde{A}+\tilde{b} \tilde{K}$ and $A+b K$ coincide). Observe that $\chi_{\tilde{A}+\tilde{b} \tilde{K}}(\lambda)=$ $\left(\lambda^{s}+\left(a_{s}-\tilde{k}_{s}\right) \lambda^{s-1}+\cdots+\left(a_{2}-\tilde{k}_{2}\right) \lambda+\left(a_{1}-\tilde{k}_{1}\right)\right) \chi_{A_{22}}(\lambda)$. Positivity of its coefficients implies that $\tilde{k}_{i}<a_{i}$ for $i=1, \ldots, s$. Since $\tilde{k}_{i}=K v_{i}$, the last inequality is equivalent to $K v_{i}<a_{i}$. Thus there must exist $K$ that satisfies (13), so the set of these inequalities is consistent.

Sufficiency. Let $K$ satisfy (13). As in the proof of Necessity, the condition $k_{j} \geq \alpha_{j}$, $j=1, \ldots, n$, means positivity of the closed-loop system $x^{\Delta}=(A+b K) x$. To show that this system is positively uniformly exponentially stable it is enough to verify that the coefficients of $\chi_{A+b K}$ are positive. As in the proof of Necessity, this is equivalent to positivity of coefficients of $\chi_{\tilde{A}+\tilde{b} \tilde{K}}$, with $\tilde{A}$ and $\tilde{b}$ given by (12) and $\tilde{K}=K T$. As before, $\chi_{\tilde{A}+\tilde{b} \tilde{K}}(\lambda)=\left(\lambda^{s}+\left(a_{s}-\tilde{k}_{s}\right) \lambda^{s-1}+\cdots+\left(a_{2}-\tilde{k}_{2}\right) \lambda+\left(a_{1}-\tilde{k}_{1}\right)\right) \chi_{A_{22}}(\lambda)$ and the condition $K v_{i}<a_{i}, i=1, \ldots, k$, implies $a_{i}-\tilde{k}_{i}>0$ for $i=1, \ldots, s$. Observe that the matrix $A_{22}$ defines the uncontrollable subsystem of the system (10), so from (11) the eigenvalues of $A_{22}$ must belong $S(\mathbb{T})$. Thus their real parts are negative, so $\chi_{A_{22}}$ has positive coefficients (see the proof of Theorem 5). Hence, $\chi_{\tilde{A}+\tilde{b} \tilde{K}}$ has positive coefficients as well.

\section{Conclusion}

We provided a characterization of uniform exponential stability of a positive linear system on a time scale. Surprisingly, regardless of the time scale, the condition is the same, which confirms usefulness of the theory and of the language of time scales. This characterization was used then to develop criteria for positive feedback stabilizability. They guarantee existence of a linear feedback that stabilizes the system and preserves its positivity. This was done for one-dimensional controls only, so a natural extension of this part would be to characterize positive stabilizability for the multi-control case. The next step could be studying stabilization of nonlinear systems via linearization.

\section{Compliance with ethical standards}

Conflict of interest The author declares that he has no conflict of interest.

Open Access This article is licensed under a Creative Commons Attribution 4.0 International License, which permits use, sharing, adaptation, distribution and reproduction in any medium or format, as long as you give appropriate credit to the original author(s) and the source, provide a link to the Creative Commons licence, and indicate if changes were made. The images or other third party material in this article are included in the article's Creative Commons licence, unless indicated otherwise in a credit line to the material. If material is not included in the article's Creative Commons licence and your intended use is not permitted by statutory regulation or exceeds the permitted use, you will need to obtain permission directly from the copyright holder. To view a copy of this licence, visit http://creativecommons.org/licenses/by/4.0/. 


\section{Appendix}

\section{Calculus on time scales}

A time scale $\mathbb{T}$ is an arbitrary nonempty closed subset of the set $\mathbb{R}$ of real numbers. In particular $\mathbb{R}, h \mathbb{Z}$ for $h>0$ and $q^{\mathbb{N}}:=\left\{q^{k}, k \in \mathbb{N}\right\}$ for $q>1$ are time scales. We assume that $\mathbb{T}$ is a topological space with the relative topology induced from $\mathbb{R}$. If $t_{0}, t_{1} \in \mathbb{T}$, then $\left[t_{0}, t_{1}\right]_{\mathbb{T}}$ denotes the intersection of the ordinary closed interval with $\mathbb{T}$. Similar notation is used for open, half-open or infinite intervals.

For $t \in \mathbb{T}$ we define the forward jump operator $\sigma_{\mathbb{T}}: \mathbb{T} \rightarrow \mathbb{T}$ by $\sigma_{\mathbb{T}}(t):=\inf \{s \in$ $\mathbb{T}: s>t\}$ if $t \neq \sup \mathbb{T}$ and $\sigma_{\mathbb{T}}(\sup \mathbb{T})=\sup \mathbb{T}$ when sup $\mathbb{T}$ is finite; the backward jump operator $\rho_{\mathbb{T}}: \mathbb{T} \rightarrow \mathbb{T}$ by $\rho_{\mathbb{T}}(t):=\sup \{s \in \mathbb{T}: s<t\}$ if $t \neq \inf \mathbb{T}$ and $\rho_{\mathbb{T}}(\inf \mathbb{T})=\inf \mathbb{T}$ when inf $\mathbb{T}$ is finite; the forward graininess function $\mu_{\mathbb{T}}: \mathbb{T} \rightarrow$ $[0, \infty)$ by $\mu_{\mathbb{T}}(t):=\sigma_{\mathbb{T}}(t)-t$; the backward graininess function $\nu_{\mathbb{T}}: \mathbb{T} \rightarrow[0, \infty)$ by $\nu_{\mathbb{T}}(t):=t-\rho_{\mathbb{T}}(t)$.

If $\sigma_{\mathbb{T}}(t)>t$, then $t$ is called right-scattered, while if $\rho_{\mathbb{T}}(t)<t$, it is called leftscattered. If $t<\sup \mathbb{T}$ and $\sigma_{\mathbb{T}}(t)=t$ then $t$ is called right-dense. If $t>\inf \mathbb{T}$ and $\rho_{\mathbb{T}}(t)=t$, then $t$ is left-dense.

The time scale $\mathbb{T}$ is homogeneous, if $\mu_{\mathbb{T}}$ and $\nu_{\mathbb{T}}$ are constant functions. When $\mu_{\mathbb{T}} \equiv 0$ and $\nu_{\mathbb{T}} \equiv 0$, then $\mathbb{T}=\mathbb{R}$ or $\mathbb{T}$ is a closed interval (in particular a half-line). When $\mu_{\mathbb{T}}$ is constant and greater than 0 , then $\mathbb{T}=\mu_{\mathbb{T}} \mathbb{Z}$.

Let $\mathbb{T}^{\kappa}:=\{t \in \mathbb{T}: t$ is nonmaximal or left-dense $\}$. Thus $\mathbb{T}^{\kappa}$ is got from $\mathbb{T}$ by removing its maximal point if this point exists and is left-scattered.

Let $f: \mathbb{T} \rightarrow \mathbb{R}$ and $t \in \mathbb{T}^{\kappa}$. The delta derivative of $f$ at $t$, denoted by $f^{\Delta}(t)$, is the real number with the property that given any $\varepsilon$ there is a neighborhood $U=$ $(t-\delta, t+\delta)_{\mathbb{T}}$ such that

$$
\left|\left(f\left(\sigma_{\mathbb{T}}(t)\right)-f(s)\right)-f^{\Delta}(t)\left(\sigma_{\mathbb{T}}(t)-s\right)\right| \leq \varepsilon\left|\sigma_{\mathbb{T}}(t)-s\right|
$$

for all $s \in U$. If $f^{\Delta}(t)$ exists, then we say that $f$ is delta differentiable at $t$. Moreover, we say that $f$ is delta differentiable on $\mathbb{T}^{k}$ provided $f^{\Delta}(t)$ exists for all $t \in \mathbb{T}^{k}$.

Example 1 If $\mathbb{T}=\mathbb{R}$, then $f^{\Delta}(t)=f^{\prime}(t)$. If $\mathbb{T}=h \mathbb{Z}$, then $f^{\Delta}(t)=\frac{f(t+h)-f(t)}{h}$. If $\mathbb{T}=q^{\mathbb{N}}$, then $f^{\Delta}(t)=\frac{f(q t)-f(t)}{(q-1) t}$.

A function $f: \mathbb{T} \rightarrow \mathbb{R}$ is called $r d$-continuous provided it is continuous at rightdense points in $\mathbb{T}$ and its left-sided limits exist (finite) at left-dense points in $\mathbb{T}$. If $f$ is continuous, then it is rd-continuous.

A function $f: \mathbb{T} \rightarrow \mathbb{R}$ is called regressive, if $1+\mu(t) f(t) \neq 0$ for all $t \in \mathbb{T}$.

A function $F: \mathbb{T} \rightarrow \mathbb{R}$ is called an antiderivative of $f: \mathbb{T} \rightarrow \mathbb{R}$ provided $F^{\Delta}(t)=f(t)$ holds for all $t \in \mathbb{T}^{\kappa}$. Let $a, b \in \mathbb{T}$. Then the delta integral of $f$ on the interval $[a, b)_{\mathbb{T}}$ is defined by

$$
\int_{a}^{b} f(\tau) \Delta \tau:=\int_{[a, b)_{\mathbb{T}}} f(\tau) \Delta \tau:=F(b)-F(a) .
$$


Riemann and Lebesgue delta integrals on time scales have been also defined (see e.g. [27]). It can be shown that every rd-continuous function has an antiderivative and its Riemann and Lebesgue integrals agree with the delta integral defined above.

Example 2 If $\mathbb{T}=\mathbb{R}$, then $\int_{a}^{b} f(\tau) \Delta \tau=\int_{a}^{b} f(\tau) d \tau$, where the integral on the right is the usual Riemann integral. If $\mathbb{T}=h \mathbb{Z}, h>0$, then $\int_{a}^{b} f(\tau) \Delta \tau=\sum_{t=\frac{a}{h}}^{\frac{b}{h}-1} f(t h) h$ for $a<b$.

\section{References}

1. Farina, L., Rinaldi, S.: Positive Linear Systems: Theory and Applications, Pure and Applied Mathematics. Wiley, New York (2000)

2. Kaczorek, T.: Positive 1D and 2D Systems. Springer, London (2002)

3. Doan, T.S., Kalauch, A., Siegmund, S.: Exponential stability of linear time-invariant systems on time scales. Nonlinear Dyn. Syst. Theory 9, 37-50 (2009)

4. Doan, T.S., Kalauch, A., Siegmund, S., Wirth, S.F.R.: Stability radii for positive linear time-invariant systems on time scales. Syst. Control Lett. 59, 173-179 (2010)

5. Davis, J.M., Gravagne, I.A., Jackson, B.J., Marks II, R.J.: Controllability, observability, realizability and stability of dynamic linear systems. Electron. J. Differ. Equ. 2009, 1-32 (2009)

6. DaCunha, J.J.: Stability for time varying linear dynamic systems on time scales. J. Comput. Appl. Math. 176, 381-410 (2005)

7. Peterson, A.C., Raffoul, Y.N.: Exponential stability of dynamic equations on time scales. Adv. Differ. Equ. 2, 133-144 (2005)

8. Liu, A.: Boundedness and exponential stability of solutions to dynamic equations on time scales. Electron. J. Differ. Equ. 12, 1-14 (2006)

9. Du, N.H., Tien, L.H.: On the exponential stability of dynamic equations on time scales. J. Math. Anal. Appl. 331, 1159-1174 (2007)

10. Bartosiewicz, Z., Piotrowska, E., Wyrwas, M.: Stability, stabilization and observers of linear control systems on time scales. In: Proceedings of the 46th IEEE Conference on Decision and Control, New Orleans, USA, December 12-14. pp. 2803-2808 (2007)

11. Davis, J.M., Gravagne, I.A., Jackson, B.J., Marks II, R.J.: State feedback stabilization of linear time-varying systems on time scales. In: 42nd South Eastern Symposium on System Theory. IEEE, University of Texas at Tyler, Tyler, March 7-9 (2010)

12. Jackson, B.J., Davis, J.M., Gravagne, I.A., Marks II, R.J.: Linear state feedback stabilization on time scales. arXiv:0910.3034v1 [math.OC] (2009)

13. Pötzsche, C., Siegmund, S., Wirth, F.: A spectral characterization of exponential stability for linear time-invariant systems on time scales. Discrete Contin. Dyn. Syst. 9, 1223-1241 (2003)

14. Martynyuk, A.A.: Stability Theory for Dynamic Equations on Time Scales. Birkhäuser, Boston (2016)

15. Grüne, L., Sontag, E.D., Wirth, F.R.: Asymptotic stability equals exponential stability, and ISS equals finite energy gain - if you twist your eyes. Syst. Control Lett. 38, 127-134 (1999)

16. Bartosiewicz, Z.: Observability of linear positive systems on time scales. In: Proceedings of the 51st IEEE Conference on Decision and Control, Maui, Hawaii, December 10-13, pp. 2581-2586 (2012)

17. Bartosiewicz, Z.: Linear positive control systems on time scales; controllability. Math. Control Signals Syst. 25, 327-343 (2013)

18. Bartosiewicz, Z.: On positive reachability of time-variant linear systems on time scales. Bull. Pol. Acad. Sci. Tech. Sci. 61, 905-910 (2013)

19. Bartosiewicz, Z.: Positive realizations on time scales. Control Cybern. 42, 315-328 (2013)

20. Bartosiewicz, Z.: Reachability and observability graphs for linear positive systems on time scales. In: Proceedings of the 19th World Congress, The International Federation of Automatic Control Cape Town, South Africa. August 24-29, pp. 3803-3808 (2014)

21. Bartosiewicz, Z.: Positive reachability and observability of linear positive time-variant systems on non-uniform time domains. In: Proceedings of the 20th World Congress The International Federation of Automatic Control, Toulouse, France, July 9-14 (2017) 
22. Bohner, M., Peterson, A.: Dynamic Equations on Time Scales: An Introduction and Applications. Birkhäuser, Boston (2001)

23. Gantmacher, F.R.: The Theory of Matrices. AMS Chelsea Publishing, New York (2000)

24. Bartosiewicz, Z., Piotrowska, E.: On stabilisability of nonlinear systems on time scales. Int. J. Control 86, 139-145 (2013)

25. Bartosiewicz, Z.: Exponential stability of nonlinear positive systems on time scales. Nonlinear Anal. Hybrid Syst. 33, 143-150 (2019)

26. Sontag, E.D.: Mathematical Control Theory: Deterministic Finite Dimensional Systems. Springer, New York (1990)

27. Guseinov, G.S.: Integration on time scales. J. Math. Anal. Appl. 285, 107-127 (2003)

Publisher's Note Springer Nature remains neutral with regard to jurisdictional claims in published maps and institutional affiliations. 